\title{
Power corrections and perturbative coupling from lattice gauge theories
}

\author{
G. Burgio ${ }^{\mathrm{a} *}$, F. Di Renzo ${ }^{\mathrm{b}}$, G. Marchesinic ${ }^{\mathrm{c}}$ and E. Onofri ${ }^{\mathrm{a}}$ \\ ${ }^{\text {a}}$ Dipartimento di Fisica, Università di Parma and INFN, Gruppo Collegato di Parma, Italy \\ ${ }^{\mathrm{b}}$ Department of Mathematical Sciences, University of Liverpool, United Kingdom \\ ${ }^{\mathrm{c}}$ Dipartimento di Fisica, Università di Milano and INFN, Sezione di Milano, Italy
}

From the analysis of the perturbative expansion of the lattice regularized gluon condensate, toghether with MC data, we present evidence of OPE-unexpected dim-2 power corrections in the scaling behaviour of the Wilson loop. These can be interpreted as an indication that in lattice gauge theories the running coupling at large momentum contains contributions of order $\Lambda^{2} / Q^{2}$.

\section{The gluon condensate}

Operator product expansion (OPE) [1] has been applied to the study of non-perturbative contributions of physical observables in asymptotically free theories [2]. In these studies an important rôle is played by the gluon condensate $\left\langle\alpha_{\mathrm{s}} \operatorname{Tr} F^{2}\right\rangle$, which, according to OPE, has the expansion

$$
W \equiv \frac{\left\langle\alpha_{\mathrm{s}} \operatorname{Tr} F^{2}\right\rangle}{Q^{4}}=W_{0}+\left(\Lambda^{4} / Q^{4}\right) W_{4}+\cdots
$$

where $\Lambda$ is the physical scale of the theory related to the running coupling $\alpha_{\mathrm{s}}=\alpha_{\mathrm{s}}\left(Q^{2}\right)$ at the scale $Q$. Since $\Lambda$ has no expansion in $\alpha_{\mathrm{s}}$, perturbative contributions are present only in $W_{0}$, which from power counting is quartically divergent in the UV region. The term with the power $\Lambda^{2} / Q^{2}$ is absent since there are no gauge invariant operators of dimension two.

In the lattice theory all frequencies are bounded by the UV cutoff $Q=\pi / a$ with $a$ the lattice spacing. The condensate $W$ can be written in the general form (we assume an infinite lattice for the moment)

$$
W=\int_{0}^{Q^{2}} \frac{k^{2} d k^{2}}{Q^{4}} f\left(k^{2} / \Lambda^{2}\right) .
$$

This expression is based on the fact that the associated observable has dimension four and is renormalization group invariant [3] so that the function

\footnotetext{
${ }^{*}$ Speaker at the conference.
}

$f\left(k^{2} / \Lambda^{2}\right)$, which does not depend on $Q$, for large $Q$, can be expressed in terms of a running coupling at the scale $k^{2}$.

In [1] it is shown that the "perturbative" contribution, obtained using for $f$ the two loop $\alpha_{\mathbf{s}}\left(k^{2}\right)$ and introducing a cut-off $\rho \Lambda^{2}$ with $\rho \gg 1$ to stay within the perturbative region, can be expressed as

$$
\begin{aligned}
W_{0}^{\text {ren }} & =\mathcal{N} \int_{0}^{z_{0}-} d z e^{-\beta z}\left(z_{0}-z\right)^{-1-\gamma} \\
& =\sum_{\ell=1} \beta^{-\ell}\left\{c_{\ell}^{\text {ren }}+\mathcal{O}\left(e^{-z_{0} \beta}\right)\right\}
\end{aligned}
$$

where the integration region is mapped into $0<$ $z<z_{0_{-}}=z_{0}(1-\bar{\beta} / \beta)$ with $6 / \bar{\beta}=4 \pi \alpha_{\mathbf{s}}\left(\rho \Lambda^{2}\right)$, $e^{-z_{0} \beta} \sim \Lambda^{4} / Q^{4}$ and $c_{\ell}^{\text {ren }}$ the renormalon coefficients

$c_{\ell}^{\mathrm{ren}}=\mathcal{N}^{\prime} \Gamma(\ell+\gamma) z_{0}^{-\ell}$.

A similar factorial growth of the perturbative coefficients is found if one considers the contributions from higher powers of the coupling. Then the expression above gives a general form of the perturbative factorial growth with the numerical constant $\mathcal{N}$ which takes into account higher order corrections.

The coefficients $c_{\ell}^{\text {lat }}(M)$ of the perturbative expansion of $W(M)$ on a lattice of size $M$

$W^{\text {pert }}(M)=\sum_{\ell \geq 1} c_{\ell}^{\text {lat }}(M) \beta_{\text {lat }}^{-\ell}$, 
are known up to eight loops. The first three terms have been computed analytically [5] for an infinite lattice. Eight terms for the expansion of both $W_{1 \times 1}$ and $W_{2 \times 2}$ have been computed numerically in Ref. [6] for a lattice with $M=8$.

In Ref. [6] it has been shown that the growth with $\ell$ of the first eight coefficients is consistent with the factorial behaviour described in the previous section for a quantity of dimension four like $W$. The analysis of finite volume effects and the comparison between continuum and lattice schemes have been performed in [4, the former by taking into account an explicit IR cutoff $Q_{0}=2 \pi / M a$ i.e. $z<z_{\text {ir }}=4 \ln (M / 2) / \beta$ in (3), the latter, in analogy with the result for $\sigma$-models [7], by assuming that the scale entering in the running coupling is $s k^{2}<k^{2}$, with $s$ such to rescale the $\Lambda$ parameter from the continuum to lattice scheme.

\section{Evidence of a $\Lambda^{2} / Q^{2}$ contribution}

Here we present the evidence that in lattice gauge theory the condensate contains terms of or$\operatorname{der} \Lambda^{2} / Q^{2} \sim e^{-z_{0} \beta / 2}$. We analyze $W-W_{0}$ as a function of $\beta$. The contribution $W$ is obtained by the Monte Carlo simulation on a $8^{4}$ lattice [8]. The contribution $W_{0}$ is constructed by adding to the computed eight-loop perturbative terms the rest of the perturbative expansion, since for large orders the perturbative coefficients of $W_{0}^{\text {ren }}(M)$ approach the lattice ones [1, 6].

In Fig. 1a we plot the quantity

$\Delta_{L} W(M)=W(M)-\sum_{\ell=1}^{L} c_{\ell}^{\text {lat }}(M) \beta_{\text {lat }}^{-\ell}$,

in the range $\beta_{\text {lat }}=6-7$ for various values of $L \leq 8$.

We observe that in this range of $\beta_{\text {lat }}$ the quantity $\Delta_{L} W(M)$ approaches for $L \rightarrow 8$ the behaviour of $\Lambda^{2} / Q^{2}$ instead than the expected behaviour of $\Lambda^{4} / Q^{4}$.

In Fig. 1b it is shown the effect of the subtraction of the perturbative expansion to all orders [4]. The behaviour $\Lambda^{2} / Q^{2}$ is mantained.

As for finite volume effects, while those on the perturbative coefficients are under control [4],
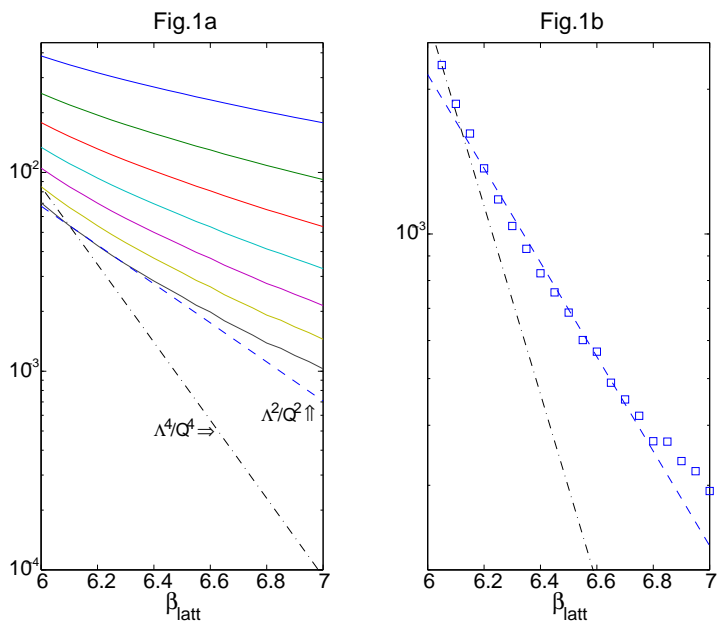

Figure 1. (a) Coefficients of the perturbative expansion as function of the (loop) order $\ell$ compared with the $\operatorname{dim}=2$ and $\operatorname{dim}=4$ scaling. (b) Subtraction of the whole resummed perturbative expansion.

those on the MC data are quite difficult to estimate without performing a direct Monte Carlo simulation on lattices with $M$ sufficient large to have the IR cutoff below the Landau singularity, i.e. $\ln (M / 2)>\beta / 12 b_{0}$.

\section{Conclusion}

We have studied the contribution $W_{0}$ obtained from the first eight terms of the perturbative expansion and a remainder constructed on the hypothesis that only $\Lambda^{4} / Q^{4}$ corrections are present. By subtracting from $W$ the term $W_{0}$ we have found indications of an additional contribution proportional to $\Lambda^{2} / Q^{2}$ (Fig. 1).

The reason for the unexpected behaviour $\Lambda^{2} / Q^{2}$ could be that the analysis is not complete. The major problem is the finiteness of the lattice size. The effects on the considered contributions to $W_{0}$ have been extimated and they seem to be small [4]. It may be that the Monte Carlo sim- 
ulation for $W$ contains spurious finite size effects giving an effective $\Lambda^{2} / Q^{2}$ behaviour. Excluding this possibility would require an investigation on a very large lattice with $\ln (M / 2) \gtrsim \beta$.

In lattice gauge theory, due to the presence of a rigid UV cutoff, the gluon condensate has no UV renormalon [9]. One may worry than that OPE could be violated. Recently it has been argued by Grunberg [10] and by Akhoury and Zakharov 11 that terms of order $\Lambda^{2} / Q^{2}$ can be present in the gluon condensate which are not accounted for by OPE, but are due to power corrections in the running coupling at high momentum. In physical schemes 12,13] highly subleading power corrections at large momentum are naturally present in the running coupling. These corrections could be responsible for the appearance of $\Lambda^{2} / Q^{2}$ terms in the condensate due to the fact that the integral for $W$ is quartically divergent. A $\Lambda^{2} / k^{2}$ contribution in $\alpha_{\mathbf{s}}\left(k^{2}\right)$ in the integral (2) gives two terms. The first of order $\Lambda^{2} / Q^{2}$ comes from the UV region $\left(k^{2} \approx Q^{2}\right)$, the second, of the canonical order $\Lambda^{4} / Q^{4}$, comes from the IR region $\left(k^{2} \approx \rho \Lambda^{2}\right)$ and mixes with the term predicted by OPE. In Fig. 2 we show a fit of the subtracted data with both contributions at the same time. The $\Lambda^{2} / Q^{2}$ terms are of "perturbative" nature and are then naturally associated to the contribution $W_{0}$ in the OPE. Moreover they should be process independent as the running coupling. An important question is whether these $\Lambda^{2} / Q^{2}$ are phenomenologically relevant (see [10,11]).

\section{REFERENCES}

1. K. Wilson, Phys. Rev. D 179 (1969) 1499.

2. M.A. Shifman, A.I. Vainstein and V.I. Zakharov, Nucl. Phys. B147 (1979) 385, 448, 519; V.A. Novikov, M.A. Shifman, A.I. Vainstein and V.I. Zakharov, Phys. Rep. 116 (1984) 105;

3. V.A. Novikov, M.A. Shifman, A.I. Vainstein and V.I. Zakharov, Nucl. Phys. B249 (1985) 445.

4. G. Burgio, F. Di Renzo, G. Marchesini and E. Onofri hep-ph/9706209, submitted to Nucl. Phys. B.

5. B. Allés, M. Campostrini, A. Feo and H.

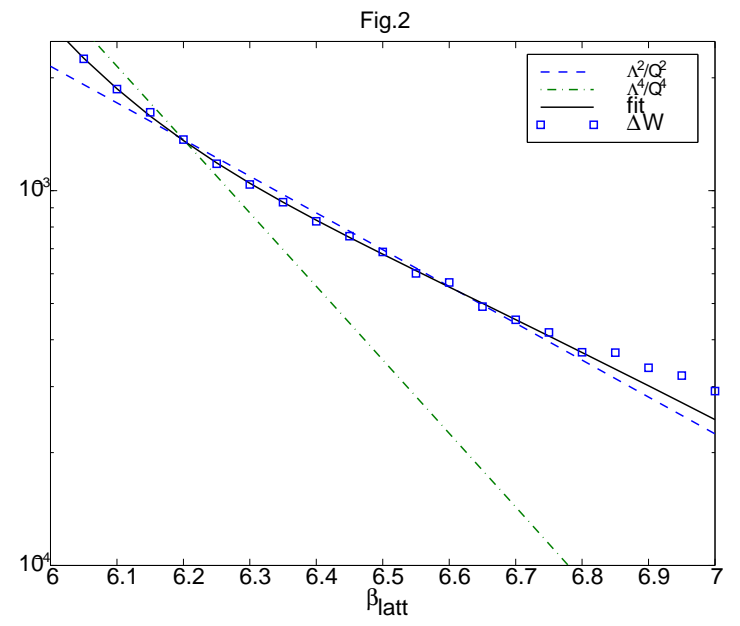

Figure 2. The subtracted data fitted with a behaviour of the type $a \Lambda^{2} / Q^{2}+b \Lambda^{4} / Q^{4}$

Panagopoulos, Phys. Lett. 324B (1994) 443 and references therein.

6. F. Di Renzo, G. Marchesini and E. Onofri, Nucl. Phys. B457 (1995) 202.

7. F. Di Renzo, G. Marchesini and E. Onofri, Nucl. Phys. B497 (1997) 435.

8. MC data from L. Scorzato (1997).

9. For reviews and classic references see: V.I. Zakharov, Nucl. Phys. B385 (1992) 452; A.H. Mueller, in $Q C D 20$ years later, vol. 1 (World Scientific, Singapore 1993).

10. G.Grunberg, hep-ph/9705290.

11. R. Akhoury and V.I. Zakharov, hepph/9705318; A.I. Vainstein and V.I. Zakharov, Phys. Rev. Lett. 73 (1994) 1207; Phys. Rev. D D54 (1996) 4039; K.K. Yamawaki and V.I. Zakharov, hep-ph/9406373.

12. Yu.L. Dokshitzer, G. Marchesini and B.R. Webber, Nucl. Phys. B469 (1996) 93

13. S.J. Brodsky, G.P. Lepage and P.B. Mackenzie, Phys. Rev. D 28 (1983) 228. 